REVISTA DE DERECHO UNED, núm. 4, 2009

\title{
FAMILIA Y SOCIEDAD ANTE LA SALUD MENTAL
}

\author{
Pedro Fernández Santiago \\ Profesor de Servicios Sociales y Política Social de la Universidad \\ Nacional de Educación a Distancia (UNED)
}

Resumen: En relación a la situación de las personas que sufren trastornos de salud mental, los pasos por dar son todavía un gran camino que entre todos tenemos que andar, si olvidadas son las personas con discapacidad en una sociedad donde lo diferente es habitualmente tenido por "raro», las personas con trastornos de salud mental son los más olvidados y apartados. Descansa fundamentalmente en las familias el gran peso de tratar patologías que en muchos casos no son diagnosticadas a tiempo, y desconocida por ellos y la mayoría de los ciudadanos en el mejor de los casos, dado que si llegan informaciones al respecto, suelen ir acompañadas de informaciones alarmantes. El desconocimiento y esta información sesgada produce que la sociedad y quien la compone (en esta, incluyo a muchas de las personas que estructuran y dirigen la función pública) rechacen y excluyan a las personas que sufren estas enfermedades. La consecuencia de todo ello, lleva a la estigmatización y posterior marginación de las mismas.

La tesis sobre la situación de marginación de las personas que sufren enfermedades de salud mental grave y duradera que defiendo, conlleva un doble proceso de marginación la que se produce por parte de la sociedad y por otro lado, la que se puede inferir por su parte, de este tipo de respuestas.

Palabras clave: Salud mental. Familia. Detección y diagnóstico. Sociedad y salud mental. Discapacidad. Rechazo. Exclusión. Marginación. 


\begin{abstract}
Abstrac: In regard to the situation of people who suffer from mental diseases there is still a long way to scour and all of us should be on the right road. If handicapped people are usually forgotten in our society, probable because it is easier to look the other way, people who suffer from mental diseases are much more isolated. The weight of treating the different pathologies lies almost completely on the family even when they are not diagnosed on time. Unknown as they are by them and by most of the citizens. Usually the information that they can get about mental diseases make them live in anxiety. This lack of information makes the society (including those who manage this kina of issue from the Government) reject people who suffer from mental diseases. The obvious consequence is branding and stigmatization.

My theory about this situation of branding and stigmatization of people who suffer long and serious mental diseases jeans a dual process of isolation: On the one hand, the one that our society brings about and on the other hand, the one that the own sick person feels us a consequence of the behaviour of the society.
\end{abstract}

Key words: mental health, family, detection and diagnosis, society and mental health, handicap, social rejection, exclusion, social isolation.

Sumario: 1. Introducción.-2. Una nueva visión jurídico social de la salud mental para nuestro país.-3. La sociedad ante las personas con enfermedad mental grave y duradera.-4. Familia y discapacidad.-5. Los procesos de marginación y estigmatización que sufren las personas con trastornos mentales graves.-6. Conclusiones.-7. Bibliografía.

\title{
1. INTRODUCCIÓN
}

La Organización Mundial de la Salud (OMS) define salud como el estado de completo bienestar físico, mental y social. La salud mental hace referencia al bienestar emocional y psicológico necesario para que el ser humano pueda utilizar sus capacidades cognitivas y emocionales, desenvolviéndose en sociedad y resolver las demandas cotidianas de la vida diaria. La salud mental suele ser entendida como el estado de equilibrio entre una persona y su entorno socio-cultural, con el fin de alcanzar dicho equilibrio se hace necesario que las personas tengan garantizada su participación en el mundo laboral, inte- 
lectual y social para así poder alcanzar su bienestar y una adecuada calidad de vida que la hagan sentirse integrada en plenitud en la sociedad donde se desarrollan como seres humanos.

La OMS ha asegurado que no existe una definición oficial acerca de salud mental ya que aparece influenciada por las diferentes culturas y la subjetividad, no obstante suele entenderse que la salud mental es un estado de bienestar emocional y psicológico relacionado, a su vez, con factores de la salud física, dado que existen evidentes conexiones entre las enfermedades mentales y biológicas. Ya en los clásicos como Platón se encuentra el reconocimiento de la salud corporal como conductor para una adecuada higiene mental, establecía específicamente que el alma «buena» por su propia excelencia, mejoraba el cuerpo en todos sus sentidos. No obstante, el Instituto de Mayores y Servicios Sociales (IMSERSO), en la Colección de Documentos Técnicos editado en el año $2007^{1}$ define la enfermedad mental grave y duradera como: Todas aquellas incluidas en el grupo de personas que padecen síntomas de psicosis funcional (típicamente diagnosticadas como esquizofrenia, trastorno bipolar pero también otras) y que como consecuencia de su enfermedad padece discapacidades persistentes.

Todas estas enfermedades, al igual que las anteriores, son resultado de factores biológicos, psicológicos y sociales. Entre los factores biológicos podemos citar los genéticos, infecciosos, traumáticos y tóxicos; los factores psicológicos personales en el curso y evolución de la enfermedad, como por ejemplo la capacidad de afrontamiento de la enfermedad por parte de quien la padece, y factores sociales y ambientales tales como la crianza, el entorno y circunstancias familiares, así como los soportes sociales para atender las situaciones de crisis que pueden condicionar la evolución.

Los griegos comprendieron la locura como una transformación del hombre en otro ser distinto mediante una fuerza proveniente de un lugar desconocido (energoumenos), idea que será asimilada por los romanos en el concepto de mente captus. Ese sentido será recogido por la tradición cristiana al identificar al enfermo como ser «poseído" por el demonio que domina su cuerpo, pero no su alma inocente, noción que llevada a sus últimas consecuencias, supuso la existencia de los procesos de brujas en los que el fuego de la hoguera

${ }^{1}$ Colección Documentos. Serie Documentos Técnicos n. 21005 (2007), Modelo de atención a las personas con enfermedad mental grave, Edt. Ministerio de Trabajo y Asuntos Sociales. Secretaria de Estado de Servicios Sociales, Familias y Discapacidad. Instituto de Mayores y Servicios Sociales (IMSERSO), Madrid. 
era concebido como la liberación del espíritu del cuerpo poseído, periodo que ocupó los siglos XV, XVI y XVII en Europa. Pero la conciencia del loco como «bestia», como animal salvaje que puede ser enjaulado y exhibido como objeto de feria permanecerá mucho más tiempo en la conciencia popular.

La práctica en el cuidado cotidiano de las personas con enfermedades mentales era: la vigilancia en el seno familiar, en ocasiones en situaciones francamente indignas; la existencia errante de aquellos que carecían de familia o no se ocupaban de él (lo que determinaba el desarraigo, los malos tratos por parte de una sociedad recelosa e incluso la expulsión de las ciudades, confiándolos a mercaderes o a barqueros); y, en ocasiones, en los casos de personas con graves trastornos de conducta, el ingreso en establecimientos carcelarios ${ }^{2}$.

\section{UNA NUEVA VISIÓN JURÍDICO-SOCIAL DE LA SALUD MENTAL PARA NUESTRO PAÍS}

Es a partir de la década de los sesenta y setenta del siglo XX cuando se produce un cierto movimiento de reforma en el seno de los servicios psiquiátricos, el impulso decisivo en nuestro país se produjo a partir de la aprobación de la Constitución de 1978, que supuso la desaparición del antiguo sistema de beneficencia, la modificación del Código Civil en materia de incapacidad y tutela por medio de la Ley 13/1983 de 24 de octubre, la intervención judicial en los internamientos involuntarios, la reforma sanitaria llevada a cabo por la Ley 14/1986 de 25 de abril, y un amplio desarrollo de materias relativas a las discapacidades psíquicas que se encuentran en la Ley 13/1982 de 7 de abril de Integración Social de Minusválidos conocida como LISMI así como en las siguientes medidas legislativas que posteriormente iremos desgranando.

Quisiera comenzar planteando algunas interrogantes sobre la materia que estamos tratando en este capitulo, ¿se consideran las enfermedades mentales graves y duraderas como patologías discapacitantes, y, a quienes las sufren como personas con discapacidad?. Sin intentar agotar al lector con definiciones, podríamos contestar afirmativamente que dichas enfermedades son discapacitantes y que las personas que las sufren son consideradas personas con discapacidad. La Ley de Integración Social de Minusválidos (LISMI) de 13/1982 de

${ }^{2}$ FERREIRÓS, C. E., «Salud mental y derechos humanos: La cuestión del tratamiento ambulatorio involuntario", Madrid, colección CERMI, n. ${ }^{\circ}$ 27, 2007, pp. 67. 


\section{7 de abril su Titulo Primero, Artículo uno menciona que:}

«Los principios que inspiran la presente Ley se fundamentan en los derechos que el artículo cuarenta y nueve de la Constitución reconoce, en razón a la dignidad que les es propia, a los disminuidos en sus capacidades físicas, psíquicas o sensoriales ${ }^{3}$ para su completa realización personal y su total integración social, y a los disminuidos profundos para la asistencia y tutela necesarias.»

El artículo dos por su parte refiere:

«El Estado español inspirará la legislación para la integración social de los disminuidos en la declaración de derechos del deficiente mental, aprobada por las Naciones Unidas el veinte de diciembre de mil novecientos setenta y uno, y en la declaración de derechos de los minusválidos, aprobada por la Resolución tres mil cuatrocientos cuarenta y siete de dicha Organización, de nueve de diciembre de mil novecientos setenta y cinco, y amoldará a ellas su actuación».

Posterior a la LISMI, como un paso para completar los derechos de las personas con Discapacidad y coincidiendo con el año internacional de la discapacidad, se aprobó la Ley 51/2003, de 2 de diciembre, de igualdad de oportunidades, no discriminación y accesibilidad universal de las personas con discapacidad (LIONDAU) La no discriminación y la accesibilidad universal son los grandes conceptos que defiende esta Ley, que en su exposición de motivos considera que:

«El concepto de accesibilidad, está en su origen muy unido al movimiento promovido por algunas organizaciones de personas con discapacidad, organismos internacionales y expertos en favor del modelo de "vida independiente", que defiende una participación más activa de estas personas en la comunidad sobre unas bases nuevas: como ciudadanos titulares de derechos; sujetos activos que ejercen el derecho a tomar decisiones sobre su propia existencia y no meros pacientes o beneficiarios de decisiones ajenas; como personas que tienen especiales dificultades para satisfacer unas necesidades que son normales, más que personas especiales con necesidades diferentes al resto de sus conciudadanos y como ciudadanos que para atender esas necesidades demandan apoyos personales, pero también modificaciones en los entornos que erradiquen aquellos obstáculos que les impiden su plena participación».

«El movimiento en favor de una vida independiente demandó en un primer momento entornos más practicables. Posteriormente, de este concepto de eliminar barreras físicas se pasó a demandar "diseño para todos", y no sólo de los entornos, reivindicando finalmente la "ac-

${ }^{3}$ En la nueva terminología empleada en la actualidad, cuando se habla de disminuidos o deficientes, se tiene que entender personas con discapacidad. 
cesibilidad universal" como condición que deben cumplir los entornos, productos y servicios para que sean comprensibles, utilizables y practicables por todas las personas. La no accesibilidad de los entornos, productos y servicios constituye, sin duda, una forma sutil pero muy eficaz de discriminación, de discriminación indirecta en este caso, pues genera una desventaja cierta a las personas con discapacidad en relación con aquellas que no lo son, al igual que ocurre cuando una norma, criterio o práctica trata menos favorablemente a una persona con discapacidad que a otra que no lo es. Convergen así las corrientes de accesibilidad y de no discriminación. Pues bien, en esta ley se recogen estas dos nuevas corrientes y confluyen con la ya antigua pero vigente LISMI, que desarrolló sobre todo medidas de acción positiva. No discriminación, acción positiva y accesibilidad universal constituyen la trama sobre la que se ha dispuesto un conjunto de disposiciones que persiguen con nuevos medios un objetivo ya conocido: garantizar y reconocer el derecho de las personas con discapacidad a la igualdad de oportunidades en todos los ámbitos de la vida política, económica, cultural y social».

Posiblemente uno de los pasos para erradicar y dotar de contenidos y recursos las necesidades de las personas en situación de dependencia y entre ellas a las personas con discapacidad, fue la aprobación de la Ley 39/2006, de 14 de noviembre de Promoción de la Autonomía Personal y Atención a las Personas en Situación de Dependencia. El Comité Español de Representantes de Personas con Discapacidad (CERMI) ${ }^{4}$ plantea que la Ley 39/2006, establece dos estrategias que son muy diferentes, que se enuncian ya en el propio título de la Ley: la promoción de la autonomía personal y la atención a las situaciones de dependencia.

De un lado, todas las personas en situación de dependencia tienen el irrenunciable derecho (sobre todo irrenunciable por parte de terceros) a que su autonomía personal alcance el nivel más elevado posible; de otro, ninguna necesidad de atención en la realización de las actividades básicas de la vida diaria puede serles regateada.

Según la definición de la Ley, las situaciones serán de mayor o menor dependencia en función de que la persona precise de ayudas más o menos frecuentes, en la realización de las actividades básicas de la vida diaria, o de que los apoyos para alcanzar su autonomía personal sean también más o menos extensos. Ambas necesidades se equiparan pues a los efectos de la Ley. Necesitar ayuda para realizar

\footnotetext{
${ }^{4}$ Declaración previa de los documentos orientativos elaborados por la Comisión de Valoración de la Discapacidad del CERMI Estatal en relación con la aplicación práctica de la Ley 39/2006.
} 
actividades básicas no es ni más ni menos grave que precisar de apoyos para alcanzar la autonomía.

En la estrategia aprobada por el Consejo Interterritorial del Sistema Nacional de Salud el 11 de diciembre de $2006^{5}$ y en el Marco normativo de las prestaciones sanitarias y sociales se establece la Cartera de servicios en salud mental del Sistema Nacional de Salud Atención primaria y se afirma que con relación a la atención a la salud mental en coordinación con los servicios de atención especializada, se debe incluir:

1. Actividades de prevención y promoción, consejo y apoyo para el mantenimiento de la salud mental en las distintas etapas del ciclo vital.

2. Detección, diagnóstico y tratamiento de trastornos adaptativos, por ansiedad y depresivos, con derivación a los servicios de salud mental en caso de quedar superada la capacidad de resolución del nivel de atención primaria.

3. Detección de conductas adictivas, de trastornos del comportamiento y de otros trastornos mentales y de reagudizaciones en trastornos ya conocidos, y, en su caso, su derivación a los servicios de salud mental.

4. Detección de psicopatologías de la infancia / adolescencia, incluidos los trastornos de conducta en general y alimentaría en particular, y derivación, en su caso, al servicio especializado correspondiente.

5. Seguimiento, de forma coordinada con los servicios de salud mental y servicios sociales, de las personas con trastorno mental grave y prolongado.

Del mismo modo y con relación a la atención especializada respecto a la Atención a la salud mental, esta debe comprender el diagnóstico y seguimiento clínico de los trastornos mentales, la psicofármacoterapia, las psicoterapias individuales, de grupo o familiares (excluyendo el psicoanálisis y la hipnosis), la terapia electroconvulsiva y, en su caso, la hospitalización.

Por su parte la atención a la salud mental, que garantizará la necesaria continuidad asistencial, debe incluir:

${ }^{5}$ Estrategia en Salud Mental del Sistema Nacional de Salud,2006, Edt. Ministerio de Sanidad y Consumo. 
1. Actuaciones preventivas y de promoción de la salud mental en coordinación con otros recursos sanitarios y no sanitarios.

2. Diagnóstico y tratamiento de trastornos mentales agudos y de las reagudizaciones de trastornos mentales crónicos, comprendiendo el tratamiento ambulatorio, las intervenciones individuales o familiares y la hospitalización cuando se precise.

3. Diagnóstico y tratamiento de trastornos mentales crónicos, incluida la atención integral a la esquizofrenia, abarcando el tratamiento ambulatorio, las intervenciones individuales y familiares y la rehabilitación.

4. Diagnóstico y tratamiento de conductas adictivas, incluidos alcoholismo y ludopatía.

5. Diagnóstico y tratamiento de los trastornos psicopatológicos de la infancia / adolescencia, incluida la atención a los niños con psicosis, autismo y con trastornos de conducta, en general, y alimentaría, en particular (anorexia / bulimia), comprendiendo el tratamiento ambulatorio, las intervenciones psicoterapéuticas en hospital de día, la hospitalización cuando se precise y el refuerzo de las conductas saludables.

6. Atención a los trastornos de salud mental derivados de las situaciones de riesgo o exclusión social.

7. Información y asesoramiento a las personas vinculadas al paciente, especialmente al cuidador/a principal.

En el mismo documento y relativo a las luces y sombras en el nuevo escenario de la salud mental de los noventa se recogen, los aspectos positivos y negativos.

\section{a) Aspectos positivos}

Hay un consenso muy general sobre aquellos aspectos en los que la reforma psiquiátrica española ha avanzado de forma muy positiva:

- Territorialización de los servicios de salud mental.

- Integración de la atención a la salud mental en la red sanitaria, consecuencia de la propia Ley de Sanidad, influyendo positivamente sobre la calidad de la atención y la propia percepción de la sociedad en general sobre la enfermedad mental. 
- Reducción importante de las camas monográficas, en favor del desarrollo de camas activas dentro de la red de servicios de salud general.

- Desarrollo de una red de atención a la salud mental homologable en todo el país.

- Formación reglada de las y los profesionales de la salud mental (psiquiatras, psicólogas y psicólogos clínicos, enfermería)

\section{b) Problemas pendientes}

El consenso no es tan general a la hora de detallar los problemas pendientes de solución:

- Insuficiencia de recursos humanos especializados en salud mental.

- Insuficientes recursos de rehabilitación y reinserción social de calidad.

- Faltan en algunos territorios programas específicos bien desarrollados para grupos especialmente vulnerables (niños y niñas $\mathrm{y}$ adolescentes, ancianos y ancianas, inmigrantes, penados $\mathrm{y}$ penadas, etc.).

- Falta una oferta más equitativa y mayor colaboración entre Comunidades Autónomas y otras Administraciones.

- Falta de una integración generalizada de la red de atención a las drogodependencias.

- Deficiencias en la organización de la información, coordinación y evaluación del sector de salud mental.

- Necesidad de una articulación más transversal y cooperativa de la red de salud mental en el ámbito del área sanitaria (servicios sociales, atención primaria, atención especializada y rehabilitación y reinserción social), que tenga en cuenta las necesidades específicas de las personas objeto de atención por este sector sanitario.

- Insuficiente desarrollo de los aspectos de prevención y de promoción de la salud mental, así como las tareas a llevar a cabo «en y con» la comunidad. 


\section{LA SOCIEDAD ANTE LAS PERSONAS CON ENFERMEDAD MENTAL GRAVE Y DURADERA}

La Convención Internacional sobre Derechos de las Personas con Discapacidad $^{6}$ es un tratado histórico ratificado por nuestro país en el año 2007 y que sitúa a la discapacidad en el plano de los derechos humanos y va a servir para dar visibilidad a una realidad que pervive en la sociedad desde el inicio de la misma. Por su importancia para el tema que tratamos mencionaremos algunos de los temas que se desarrollan en su articulado relativo a la salud mental grave y duradera, así como a las personas que la sufren.

Ya en su Artículo 1. se afirma la necesidad de promover, proteger y asegurar el goce pleno y en condiciones de igualdad de todos los derechos humanos y libertades fundamentales por todas las personas con discapacidad, y promover el respeto de su dignidad inherente, así como que entre las personas con discapacidad se incluyen a todas aquellas que tengan deficiencias físicas, mentales, intelectuales o sensoriales a largo plazo y que, al interactuar con diversas barreras, puedan impedir su participación plena y efectiva en la sociedad, en igualdad de condiciones con las demás.

Es en el Artículo 2 de dicha Convención Internacional en el que se producen las definiciones destacaremos por la importancia de lo defendido en este capitulo, lo que se define y entiende por discriminación:

«Por discriminación por motivos de discapacidad se entenderá cualquier distinción, exclusión, o restricción por motivos de discapacidad que tenga el propósito o el efecto de obstaculizar o dejar sin efecto el reconocimiento, goce o ejercicio, en igualdad de condiciones, de todos los derechos humanos y libertades fundamentales en los ámbitos político, económico, social, cultural, civil o de otro tipo. Incluye todas las formas de discriminación, entre ellas, la denegación de ajustes razonables».

Los principios generales en los que se basa dicha Convención y que se contemplan en el artículo 3, son: El respeto de la dignidad inherente, la autonomía individual, incluida la libertad de tomar las propias decisiones, y la independencia de la persona; la no discrimi-

${ }^{6}$ Texto de conformidad con el Informe final del Comité Especial encargado de preparar una convención internacional amplia e integral para proteger y promover los derechos y la dignidad de las personas con discapacidad, presentado por el Secretario General a la Asamblea General, de conformidad con la resolución 60/232 de la Asamblea, Documento descargado de http://www.un.org/esa/socde/enable. 
nación; la participación e inclusión plenas y efectivas en la sociedad; el respeto por la diferencia y la aceptación de las personas con discapacidad como parte de la diversidad y la condición humana; la igualdad de oportunidades; la accesibilidad; la igualdad entre el hombre y la mujer así como el respeto a la evolución de las facultades de los niños y niñas con discapacidad y de su derecho a preservar su identidad.

Por su parte y en el artículo 8 y con relación a la «Toma de Conciencia» se plantea la necesidad que los Estados Parte se comprometan a adoptar medidas inmediatas, efectivas y pertinentes para que se sensibilice a la sociedad, incluso en el ámbito familiar, para que tome mayor conciencia respecto de las personas con discapacidad y fomentar el respeto de los derechos y la dignidad de estas personas; se luche contra los estereotipos, los prejuicios y las prácticas nocivas respecto de las personas con discapacidad, incluidos los que se basan en el género o la edad, en todos los ámbitos de la vida y del mismo modo promover la toma de conciencia respecto de las capacidades y aportaciones de las personas con discapacidad.

El Artículo 19 por su parte recoge el Derecho a vivir de forma independiente y a ser incluido en la comunidad, e insta a Los Estados Partes en la presente Convención a reconocer el derecho in igualdad de condiciones de todas las personas con discapacidad a vivir en la comunidad, con opciones iguales a las de los demás, y adoptarán medidas efectivas y pertinentes para facilitar el pleno goce de este derecho por las personas con discapacidad y su plena inclusión y participación en la comunidad, asegurando en especial que: Las personas con discapacidad deben tener la oportunidad de elegir su lugar de residencia y dónde y con quién vivir, en igualdad de condiciones con las demás, y no se vean obligadas a vivir con arreglo a un sistema de vida especifico; el acceso a una variedad de servicios de asistencia domiciliaria, residencial y otros servicios de apoyo de la comunidad, incluida la asistencia personal que sea necesaria para facilitar su existencia y su inclusión en la comunidad y para evitar su aislamiento o separación de ésta y a las instalaciones y los servicios comunitarios para la población en general estén a disposición, en igualdad de condiciones, de las personas con discapacidad y del mismo modo tengan en cuenta sus necesidades.

En el Artículo 27 y en relación con el Trabajo y empleo se insta a los Estados parte a reconocer el derecho de las personas con discapacidad a trabajar, en igualdad de condiciones con las demás; ello incluye el derecho a tener la oportunidad de ganarse la vida mediante 
un trabajo libremente elegido o aceptado en un mercado y un entorno laborales que sean abiertos, inclusivos y accesibles a las personas con discapacidad. Los Estados Partes salvaguardarán y promoverán el ejercicio del derecho al trabajo, incluso para las personas que adquieran una discapacidad durante el empleo, adoptando medidas pertinentes, incluida la promulgación de legislación.

Después de este repaso sobre los derechos de las personas con discapacidad y como no podía ser de otro modo incluyendo entre ellas a las personas con enfermedad mental grave y duradera, quisiera destacar que uno de los graves problemas con los que nos encontramos con relación a las personas con discapacidad es la invisibilidad a la que están sujetos, invisibilidad consecuencia en gran medida por la incapacidad de esta sociedad de presentar la diversidad humana como una parte "positiva» de la misma, la diferencia nos hace humanos, la uniformidad nos hace máquinas; ni la raza, el sexo, la ideología, la condición sexual, etcétera, nos hace ni mejores ni peores, ni siquiera diferentes, dado que en ninguno de los casos dejamos de ser humanos y sujetos a los mismos derechos y deberes como tales. Cuando dejamos de ver a los demás conciudadanos como a seres humanos con nuestros mismos derechos, la consecuencia inmediata es la sensación de que no existen y si existieran podrían llegar incluso a ser una amenaza, si no se les ve puede parecer que dejan de existir. Otra de las consecuencias de esta invisibilidad es la inadaptación de la sociedad a sus necesidades, se están produciendo grandes avances en la accesibilidad y el diseño para todos, no obstante las personas con trastornos mentales siguen siendo en gran parte los más olvidados de entre los olvidados.

Quisiera en este epígrafe plantear algunas interrogantes como: ¿Podemos negarle a una persona que sufre un trastorno bipolar diagnosticado, y que está bajo tratamiento farmacológico y estable psicológicamente, un puesto de trabajo?, ¿Podríamos hacer lo mismo con una persona que ha sufrido, pongamos por caso, una operación de corazón con el fin de incorporarle una válvula en el mismo?, ¿Podemos rechazar como trabajador a una persona insulinodependiente?

Con el fin de dar contestación a estas preguntas de forma realista diría que: según las condiciones medioambientales y de seguridad del puesto de trabajo, si estas estuvieran adaptadas al trabajador, y este tuviera los conocimientos y habilidades para su desarrollo, sin ningún genero de dudas mi contestación seria afirmativa, dado que las personas que sufren trastornos mentales graves y duraderos, y que están 
diagnosticados y tratados farmacológicamente pueden y deben desarrollar adecuadamente cualquier actividad de la vida diaria incluido el trabajo para el que estén cualificados, del mismo modo estos pueden y son considerados personas con discapacidad, y esta consideración debe y puede posibilitar que los procesos de integración e inserción sociolaboral sean afines y ajustados al resto de las personas con discapacidad. Siendo amparados por los derechos de todos los ciudadanos y los que se desarrollan para conseguir la plena integración de las personas con discapacidad en nuestro país.

En muchas ocasiones he repetido (creo que sigue siendo necesario) que la discapacidad es considerada como un «totum revolutum» al que se incorporan las múltiples patologías que pueden hacer definir a un ser humano como persona con discapacidad. Las políticas de integración social de personas con discapacidad y las diversas legislaciones al respecto están avanzando y siendo constantemente cuestionadas para que cumplan efectivamente con lo estipulado en sus articulados. Se facilitan ayudas para la contratación, se elaboran y diseñan políticas de accesibilidad, comienzan a elaborarse campañas de concienciación sobre algunas de las discapacidades en los medios de comunicación; en la Universidad comenzamos a diseñar e impartir Jornadas, Cursos de Expertos y Master relativos a algunas de las discapacidades, etcétera. Un buen comienzo se podría afirmar pero todavía queda mucho por hacer, el primer paso es hacer que cale en la opinión publica y en la sociedad en general la necesidad de que se apliquen y cumplan los preceptos que contiene nuestra Constitución.

La Organización Mundial de la Salud (OMS), la Comisión Europea y el Consejo de Europa para debatir la situación de la salud mental en Europa celebraron una Conferencia Ministerial en Helsinki (Finlandia) del 12 al 15 de enero de 2005. Basándose en el informe mundial de la salud de la OMS 2001 y en anteriores resoluciones de la Unión Europea, se reconoce que la salud mental es fundamental para la calidad de vida y productividad de las personas, las familias, la comunidad y los países, y se establece la importancia y la urgencia de afrontar los retos actuales y ofrecer soluciones basadas en la evidencia. En el se reconoce que la salud mental es fundamental para la calidad de vida y productividad de las personas, comunidades y países. A su vez se presentó el Plan de Acción en Salud Mental para Europa que los Ministros de Salud de la Región Europea de la OMS se comprometieron a respaldar, dicho Plan está enmarcado para un período de diez años y en ellos se propone: 
1. Promover el bienestar mental para todos.

2. Demostrar la importancia central de la salud mental para conseguir una buena salud pública.

3. Rechazar el estigma y la discriminación.

4. Promover actividades de sensibilización en las etapas vulnerables de la vida.

5. Prevenir los problemas de salud mental y suicidio.

6. Asegurar el acceso adecuado de los problemas de salud mental a la atención primaria de salud.

7. Ofrecer cuidados efectivos en servicios comunitarios para personas con problemas severos de salud mental.

8. Establecer coordinación entre los sectores.

9. Crear suficientes y competentes profesionales.

10. Establecer buena información en salud mental.

11. Proveer fondos equitativos y adecuados.

12. Evaluar la efectividad y generar nuevas evidencias.

Del mismo modo, se comprometieron a hacer frente a estos desafíos apoyándose en los siguientes pilares:

1. Preparar políticas e implementar actividades en contra del estigma y la discriminación y promover el bienestar mental con la inclusión de hábitos saludables en las escuelas y lugares de trabajo.

2. Determinar el impacto de salud mental en la política pública.

3. Incluir la prevención de problemas de salud mental y de suicidio en políticas nacionales.

4. Desarrollar servicios especializados capaces de dirigir cambios específicos en los jóvenes, los ancianos y en problemas de género.

5. Priorizar servicios cuyo objetivo sean los problemas de salud mental, de marginalidad y los grupos vulnerables.

6. Desarrollar asociaciones que trabajen de forma intersectorial.

7. Aumentar el número y la competencia de profesionales dedicados a salud mental. 
8. Definir, en asociación con otros Estados Miembros, el grupo de indicadores de salud mental y determinantes epidemiológicos para realizar el diseño y distribución de servicios.

9. Asegurar la financiación y consolidar la regulación y legislación equitativa de la salud incluida la salud mental.

10. Finalizar con los tratamientos y cuidados inhumanos y degradantes, promulgando los derechos humanos y la legislación de salud mental, ajustándose a las normas de la Convención de las Naciones Unidas y la legislación internacional.

11. Incrementar el grado de integración social de las personas con problemas de salud mental.

12. Asegurar la representación de usuarios y cuidadores en comités y grupos responsables de planificar, distribuir, analizar e inspeccionar las actividades de salud mental.

\section{FAMILIA Y DISCAPACIDAD}

Tal vez ser padres sea una tarea ardua y cargada de responsabilidades e incertidumbres, pero es, y debería ser, una elección libre y responsable, si partimos de la responsabilidad que debemos asumir como tales y a la vez enfrentarnos a hijos e hijas con alguna discapacidad, la tarea de ser padres puede ser como una gran y pesada losa ante la incertidumbre sobre el futuro de nuestros hijos cuando nosotros dejemos de poder prestarles el apoyo necesario, para intentar articular una explicación para este punto seguiré en gran parte el epígrafe que con el mismo titulo se encuentra publicado en el libro «Los menores con discapacidad en España» ${ }^{7}$.

El artículo 154 del Código Civil español, establece por una parte que: «la patria potestad se ejercerá siempre en beneficio de los hijos, de acuerdo con su personalidad» y por otra, que: «si los hijos tuvieren suficiente juicio deberán ser oídos siempre antes de adoptar decisiones que les afecten», en el mismo artículo se establecen una serie de deberes que los padres han de cumplir para la satisfacción del derecho del niño como es «velar por ellos, tenerlos en su compañía, alimentarlos, educarlos y procurarles una formación integral».

${ }^{7}$ FERNÁNDEZ SANTIAGO, P., «Infancia y discapacidad. Las organizaciones de personas con discapacidad, en «Los menores con discapacidad en España» CERMIUNED, Madrid, Cinca, 2008, p. 125-146. 
Para sobrevivir los seres humanos necesitan de la ayuda de los adultos durante un período de tiempo relativamente largo. Este hecho ha llevado a todas las sociedades a organizarse en torno a grupos de personas que generalmente conocemos bajo el nombre de familia. Dada la ingente cantidad de modalidades que los diferentes grupos humanos han inventado para organizar su convivencia, podríamos afirmar que la familia se está constantemente redefiniendo,

«la familia es un grupo humano de especial importancia para el desarrollo psicosocial de los niños. Las relaciones y los vínculos que se establecen durante la infancia u la adolescencia dentro del grupo familiar van a influir en gran medida en el desarrollo y calidad de los lazos afectivos que se generen y mantengan a lo largo de la vida (...) En la familia confluyen individuos con características propias que van evolucionando tanto en su dimensión de seres independientes, como en su faceta de miembros de la misma. En este sentido, la familia constituye un contexto de socialización y de desarrollo no sólo para los hijos sino también para los padres» (Martínez Borda, 2007) .

Por otra parte y siguiendo con la familia recogemos una idea que nos parece importante

«El ideal de familia nuclear cerrada se ha desmoronado; sin embargo, esto no significa necesariamente una pérdida del rol de la familia y de sus vínculos. Las relaciones de parentesco, lejos de dejar de existir, parece que toman nuevas fuerzas y se convierten en un valor sólido» (Iglesias de Ussel, 1998)

Vivir con la familia implica vivir con los padres y hermanos, si los hubiera, con el fin último de conseguir el libre y pleno desarrollo de la personalidad del niño, por la relevancia del tema mencionaremos el contenido de las relaciones paterno filiales que contiene el artículo 154 de nuestro Código Civil: Los hijos no emancipados están bajo la potestad de los padres. La patria potestad se ejercerá siempre en beneficio de los hijos, de acuerdo con su personalidad, y con respeto a su integridad física y psicológica. Esta potestad comprende los siguientes deberes y facultades:

1. Velar por ellos, tenerlos en su compañía, alimentarlos, educarlos y procurarles una formación integral

2. Representarlos y administrar sus bienes.

${ }^{8}$ MARTÍNEZ, B., «El niño y su familia desde una perspectiva psicosocial», en BARRANCO, M. ${ }^{\mathrm{a}} \mathrm{C}$, GARRIDO, M. ${ }^{\mathrm{a}}$ y GUILLO, $\mathrm{J}$ «El Derecho del niño a vivir en su propia familia»; Madrid, Exilibris, 2007.

${ }^{9}$ IGLESIAS DE USSEL, J., "La familia y el cambio político en España». Madrid, Tecnos, 1998, pp. 76. 
Si los hijos tuvieren suficiente juicio deberán ser oídos siempre antes de adoptar decisiones que les afecten. Los padres podrán, en el ejercicio de su potestad, recabar el auxilio de la autoridad.

En muchos momentos cuando estudiamos, analizamos e intervenimos en los campos de la discapacidad y la familia nos encontramos con la angustia, el miedo y la incertidumbre que en muchos casos sienten los padres de los hijos con discapacidad, debido a la inseguridad que les produce el futuro de sus hijos cuando ellos falten. Cumplir con las obligaciones naturales, morales y culturales de ser padres se hace complejo cuando no se cuenta con los adecuados recursos para alcanzar tal fin.

En los países de nuestro entorno, así como en el nuestro, contamos, como estamos viendo, con medidas legislativas que nos marcan líneas legales de obligado cumplimiento, es fácil comprender que sin la adopción de otras medidas de apoyo económico y social a la familia muchos de nuestros hijos quedarían desprotegidos, no sólo por el nivel de renta, sino también por la ausencia de recursos adaptados adecuados, desigualitaria asignación de recursos entre las diferentes comunidades sean estas rurales o urbanas, diferenciación de rentas en función del lugar de residencia, desajustes económicos imprevisibles, y un largo etcétera

La participación y la ciudadanía activa suponen que se tenga el derecho, los medios y la posibilidad de intervenir en las decisiones y de actuar para contribuir a la edificación o construcción de una sociedad mejor, y que se dispone del marco y, si procede, de la ayuda necesaria para hacerlo. Las organizaciones de jóvenes con discapacidad deberían ser consultadas en el momento de la elaboración de las políticas y programas de la juventud. Los jóvenes con discapacidad deberían poder hacer oír su voz en todos los expedientes que les afecten. Los jóvenes con discapacidad se encuentran también con obstáculos considerables en todos los ámbitos de su vida: educación, empleo, deporte, cultura, ocio y vida social. Únicamente una estrategia global, permitirá encontrar soluciones a estos problemas. Durante la elaboración de cualquier política de la juventud es indispensable buscar los medios que garanticen su plena participación en la sociedad, habida cuenta de sus necesidades especiales. Como subraya la Carta Europea sobre la participación de los jóvenes en la vida local y regional, la participación activa de éstos en las decisiones y acciones a nivel local y regional es fundamental si queremos construir sociedades más democráticas, más solidarias y más prósperas. 


\section{LOS PROCESOS DE MARGINACIÓN Y ESTIGMATIZACIÓN QUE SUFREN LAS PERSONAS CON TRASTORNOS MENTALES GRAVES}

Parto de la tesis en la que afirmo que las personas que sufren trastornos mentales graves y duraderos sufren un alto grado de estigmatización y como consecuencia marginación. Esto se manifiesta por dos vías fundamentales:

1. Respuesta de la sociedad ante los enfermos mentales

2. Respuesta de los enfermos mentales ante la sociedad

\section{Respuesta de la sociedad ante los enfermos mentales}

Las enfermedades mentales graves y duraderas son todavía en la actualidad poco conocidas, como consecuencia de esto se tarda en diagnosticar dichas patologías muchos años, en algunos de los casos estas personas son consideradas personas «raras» o "pintorescas» pero no enfermas, este desconocimiento alcanza incluso a los profesionales clínicos encargados de diagnosticar y tratar dichas enfermedades. En una de las entrevistas realizadas recientemente, y en relación con esta última afirmación, comentaba un paciente que visita a su psiquiatra acompañado por un amigo, que se dirige a él afirmando: «Tú eres Dios, yo soy Jesucristo, y éste (por el amigo) es el Espíritu Santo» La respuesta, por otra parte actualmente no habitual del profesional de la salud mental, fue «que se fuera a casa», el amigo al salir de la consulta le acompañó al hospital donde le ingresaron y trataron durante un largo período de días. Las razones para que se produzca un inadecuado diagnóstico es, entre otras muchas, que determinadas patologías mentales pueden estar enmascaradas y aparecer cuando se está siendo tratado para otra de las manifestaciones clínicas de la misma. Otra de las razones que esgrimen las personas que sufren de estas enfermedades es que para los profesionales de la salud mental es «difícil» realizar un adecuado diagnostico dado que se conoce todavía muy poco sobre las mismas. Estas dificultades en profesionales cualificados para el diagnóstico y tratamiento se hacen en muchos de los casos insalvables para el conjunto de la población, como consecuencia de ello se produce un efecto de estigmatización y marginación que intento reflejar en el grafico siguiente: 


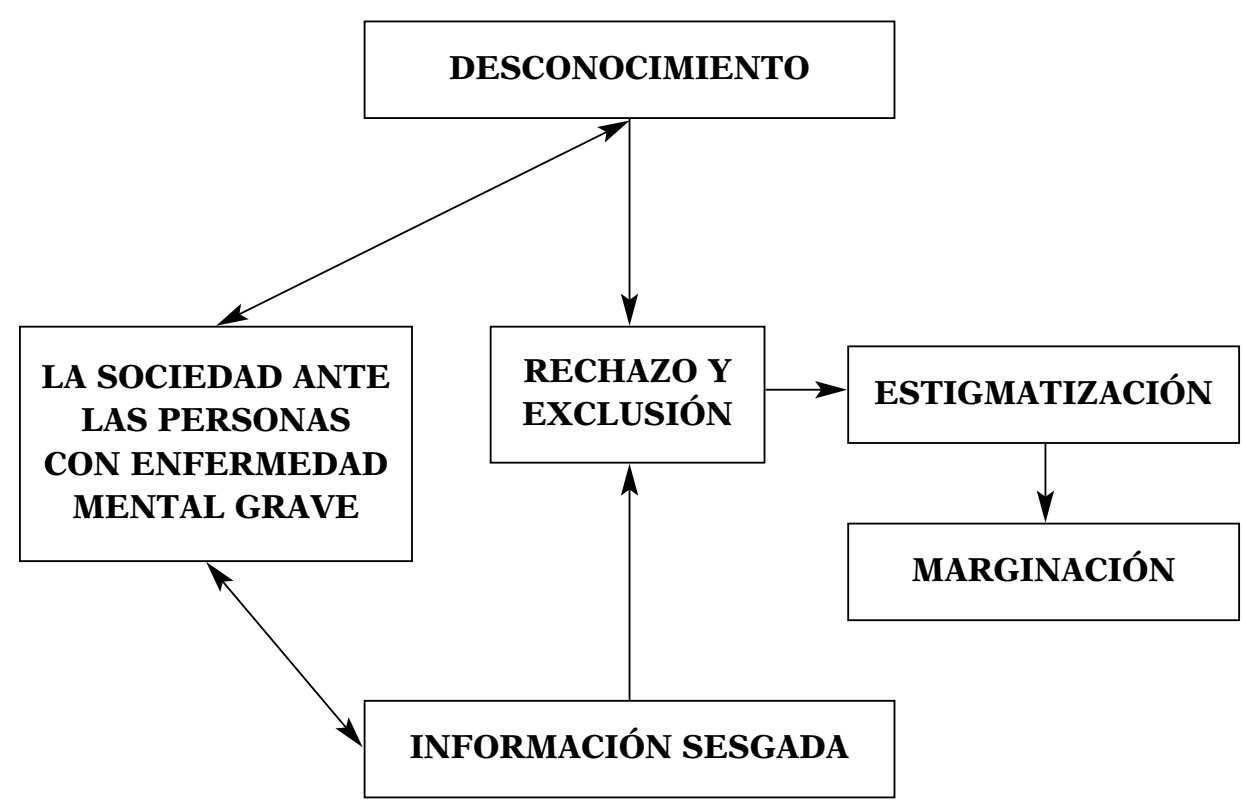

Fuente: Elaboración propia.

Entiendo por exclusión, el efecto de excluir y a este último término como efecto de dejar de aplicarle el mismo trato que a las demás personas llegando incluso a situaciones como: apartar, descartar, eliminar, exceptuar y suprimir. Marginar como: dejar en situación de inferioridad a determinados grupos humanos en relación con el resto de la sociedad, y Estigma como acto o circunstancia que constituye una deshonra para alguien y así mismo estigmatizar como dejar a alguien marcado con una imputación infamante.

Como estamos intentando reflejar en el grafico adjunto, la sociedad desconoce la situación de las personas que sufren del amplio catalogo de enfermedades mentales, desconoce las causas, los efectos, las circunstancias, etcétera. A su vez reciben informaciones en algunos momentos totalmente sesgadas de éstas ¡la información alarmante es la que vende! El desconocimiento y esta información sesgada produce que la sociedad y quien la compone (en esta, incluyo a muchas de las personas que estructuran y dirigen la función pública) rechacen y excluyan a las personas que sufren estas enfermedades. La consecuencia de todo ello, lleva a la estigmatización y posterior marginación de las mismas. 


\section{Respuesta de la persona que sufre enfermedad mental ante la estigmatización y marginación social}

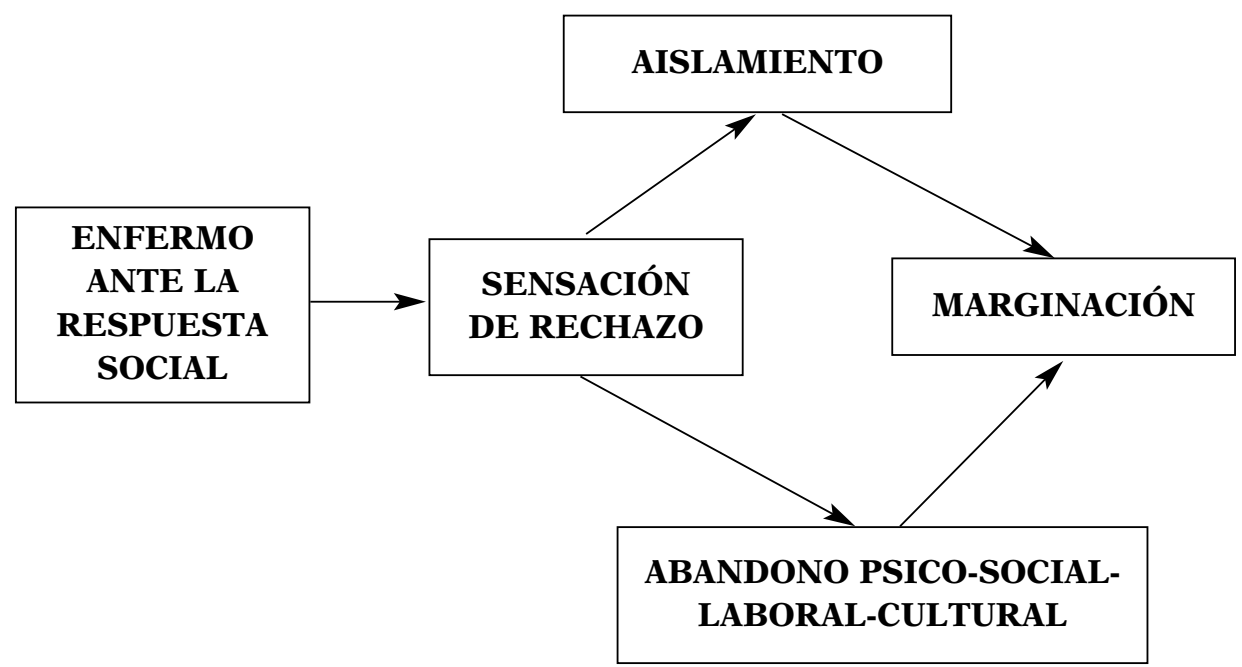

Fuente: Elaboración propia.

Las personas que sufren trastornos mentales graves y duraderos, ante la respuesta social a su enfermedad en muchos de los casos no sólo contraproducente sino incentivadora de mantenimiento y difícil tratamiento y de esa constante y continua muestra de rechazo, responden con un abandono de los aspectos psicológicos, sociales, laborales y de ocio-culturales; situación que les encamina hacia el aislamiento y como consecuencia marginación social

La tesis sobre la situación de marginación que defiendo como vemos conlleva un doble proceso de marginación sobre estas personas por un lado la que se produce por parte de la sociedad y por otro lado la que se puede inferir por su parte, de este tipo de respuestas.

\section{CONCLUSIONES}

Se están realizando numerosos esfuerzos por armonizar los derechos sociales de las personas pertenecientes a los colectivos más desfavorecidos. Son tareas que se ven claramente reflejadas en la vida cotidiana: transportes públicos adaptados; una Administración al alcance de todos sean cuales fueren sus condiciones físicas, psíquicas 
o sensoriales; obligatoriedad de adaptación a dichas circunstancias en las nuevas edificaciones, lucha por la no-discriminación laboral de las personas con discapacidad, etcétera.

La situación de las mujeres se ha comparado con la de los hombres, la discapacidad con la no discapacidad; observamos el trabajo, la salud, la familia y la violencia con una visión cuantitativa que esconde la realidad y el mundo de las personas con discapacidad. Con esto reducimos la cuestión de la discapacidad a algo meramente orgánico. Desde esta perspectiva, se hacen cada día más necesarios la elaboración y el diseño de nuevos indicadores que abarquen con amplitud la realidad de la discapacidad, así como el abordaje multidisciplinar de la misma.

Nuestra Carta Magna, así como las diferentes aportaciones legislativas que nuestro país ha desarrollado en materia de discapacidad y dependencia y, la ratificación de las diferentes Convenciones Internacionales sobre derechos humanos y discapacidad ratificadas, hace que dispongamos de un acervo legislativo importante en dicha materia, no obstante una cuestión es contar con ello y otra bien diferente es su aplicación y puesta en marcha, un ejemplo de ello, podría ser la dificultad que estamos encontrando para la adecuada puesta en marcha de la Ley de promoción de la autonomía personal y atención a las personas en situación de dependencia, con esto queremos decir que: contamos con medidas legales, pero dificultades competenciales y presupuestarias en su aplicación, como algunos de los representantes de las personas con discapacidad refieren; «una cosa es querer y otra bien diferentes es poder».

En relación a la situación de las personas que sufren trastornos de salud mental, los pasos por dar son todavía un gran camino que entre todos tenemos que andar, si olvidadas son las personas con discapacidad en una sociedad donde lo diferente es habitualmente tenido por «raro», las personas con trastornos de salud mental son los más olvidados y apartados. Descansa fundamentalmente en las familias el gran peso de tratar patologías que en muchos casos no son diagnosticadas a tiempo, y desconocida por ellos y la mayoría de los ciudadanos en el mejor de los casos, dado que si llegan informaciones al respecto, suelen ir acompañadas de informaciones alarmantes. El desconocimiento y esta información sesgada produce que la sociedad y quien la compone (en esta, incluyo a muchas de las personas que estructuran y dirigen la función pública) rechacen y excluyan a las personas que sufren estas enfermedades. La consecuencia de todo ello, lleva a la estigmatización y posterior marginación de las mismas. 
La tesis sobre la situación de marginación de las personas que sufren enfermedades de salud mental grave y duradera que defiendo, conlleva un doble proceso de marginación la que se produce por parte de la sociedad y por otro lado la que se puede inferir por su parte, de este tipo de respuestas.

Como debería traslucirse de estas paginas, la voluntad de intervención en materia de discapacidad por parte del legislador en la actualidad es incuestionable, existe una clara voluntad por integrar y cumplir y hacer cumplir las leyes que para tal fin se aprueban, otra cosa bien diferentes es que la aplicación de esta voluntad cumpla con las necesidades reales de la población a la que va dirigida, tal vez y es importante que se resalte, no se cuenta con la población a las que se dirigen las medidas y cuando si se hace, los cambios que estos proponen conllevan arduas negociaciones en las que por el camino se abandonan algunas medidas que por su importancia no deberían dejarse de lado. Prioridades, oportunidad, coyuntura política, presupuestos y un largo etcétera suelen ser los argumentos que se arguyen.

Los conceptos de prioridad, oportunidad, circunstancias favorables, momento económico, situación política favorable, y un largo etcétera, importunan la adecuada puesta en marcha de medidas necesariamente globalizadoras de las necesidades de quien se quiere atender. Está claro que toda aplicación legislativa en materia de política social conlleva un gasto público, legislar sin contar previamente con la carga impositiva o recursos económicos necesarios es «un canto al sol». Puede parecer que la visión del legislador no cumpla la necesaria visión globalizadora y se dirija al más amplio espectro de la población y no al total de la misma, esto parece desprenderse de las inajustadas medidas compensatorias entre población rural y urbana y las derivadas de la necesaria supervisión y evaluación de resultados e inversión entre las diferentes Comunidades Autónomas de nuestro país.

\section{BIBLIOGRAFÍA}

FERNÁNDEZ, P., «Infancia y discapacidad. Las organizaciones de personas con discapacidad», en "Los menores con discapacidad en España» CERMI-UNED, Madrid, Cinca, 2008, p. 125-146

FERREIRÓS, C. E. «Salud mental y derechos humanos: La cuestión del tratamiento ambulatorio involuntario».Madrid, Colección CERMI, n. ${ }^{\circ} 27,2007$ 
IGLESIAS DE USSEL, J.. «La familia y el cambio político en España». Madrid, Tecnos, 1998, p. 76

MARTÍNEZ, R. (2007), El niño y su familia desde una perspectiva psicosocial, en BARRANCO, M. ${ }^{a} \mathrm{C}$; GARRIDO Y GUILLO, $\mathrm{J}$ «El Derecho del niño a vivir en su propia familia»; Madrid, Exilibris, 2007.

\section{DOCUMENTOS}

Colección Documentos. Serie Documentos Técnicos n. 21005 (2007), Modelo de atención a las personas con enfermedad mental grave, Ministerio de Trabajo y Asuntos Sociales. Secretaria de Estado de Servicios Sociales, Familias y Discapacidad. Instituto de Mayores y Servicios Sociales (IMSERSO).

Estrategia en Salud Mental del Sistema Nacional de Salud (2006), Ministerio de Sanidad y Consumo. 